\title{
Physiologic Monitor Alarms for Children: Pushing the Limits
}

\author{
Christopher P. Bonafide, MD, MSCE ${ }^{1,2 *}$, Patrick W. Brady, MD, MSc ${ }^{3,4}$, Carrie Daymont, MD, MSCE 5,6
}

\begin{abstract}
'Division of General Pediatrics, The Children's Hospital of Philadelphia, Philadelphia, Pennsylvania; ${ }^{2}$ Perelman School of Medicine at the University of Pennsylvania, Philadelphia, Pennsylvania; ${ }^{3}$ Division of Hospital Medicine, Cincinnati Children's Hospital Medical Center, Cincinnati, Ohio; ${ }^{4}$ Department of Pediatrics, University of Cincinnati College of Medicine, Cincinnati, Ohio; ${ }^{5}$ Children's Hospital Research Institute of Manitoba, Winnipeg, Manitoba, Canada; ${ }^{6}$ Department of Pediatrics and Child Health, University of Manitoba, Winnipeg, Manitoba, Canada.
\end{abstract}

Deciding when a hospitalized child's vital signs are acceptably within range and when they should generate alerts, alarms, and escalations of care is critically important yet surprisingly complicated. Many patients in the hospital who are recovering appropriately exhibit vital signs that fall outside normal ranges for well children. In a technology-focused hospital environment, these out-of-range vital signs often generate alerts in the electronic health record (EHR) and alarms on physiologic monitors that can disrupt patients' sleep, generate concern in parents, lead to unnecessary testing and treatment by physicians, interrupt nurses during important patient care tasks, and lead to alarm fatigue. It is this last area, the problem of alarm fatigue, that Goel and colleagues ${ }^{1}$ have used to frame the rationale and results of their study reported in this issue of the Journal of Hospital Medicine.

Goel and colleagues correctly point out that physiologic monitor alarm rates are high in children's hospitals, and alarms warranting intervention or action are rare. ${ }^{2-6}$ Few studies have rigorously examined interventions to reduce unnecessary hospital physiologic monitor alarms, especially in pediatric settings. Of all the potential interventions, widening parameters has the most face validity: if you set wide enough alarm parameters, fewer alarms will be triggered. However, it comes with a potential safety tradeoff of missed actionable alarms.

Before EHR data became widely available for research, normal (or perhaps more appropriate for the hospital setting, "expected") vital sign ranges were defined using expert opinion. The first publication describing the distribution of EHR-documented vital signs in hospitalized children was published in $2013 .^{7}$ Goel and colleagues have built upon this prior work in their article, in which they present percentiles of EHR-documented heart rate (HR) and respiratory rate (RR) developed using data from more than 7000 children hospitalized at an academic children's hospital.

*Address for correspondence and reprint requests: Christopher $\mathrm{P}$. Bonafide, MD, MSCE, Division of General Pediatrics, The Children's Hospital of Philadelphia, 3401 Civic Center Blvd., Philadelphia, PA 19104; Telephone: 267-426-2901; E-mail: bonafide@email.chop.edu

Received: June 13, 2016; Accepted: June 15, 2016 2016 Society of Hospital Medicine DOI 10.1002/jhm.2638 Published online in Wiley Online Library (Wileyonlinelibrary.com).
In a separate validation dataset, they then compared the performance of their proposed physiologic monitor alarm parameters-the 5th and 95th percentiles for HR and RR from this study-to the 2004 National Institutes of Health (NIH) vital sign reference ranges ${ }^{8}$ that were the basis of default alarm parameters at their hospital. They also compared their percentiles to the 2013 study.

The 2 main findings of Goel and colleagues' study were: (1) using their separate validation dataset, $55.6 \%$ fewer HR and RR observations were out of range based on their newly developed percentiles as compared to the NIH vital sign reference ranges; and (2) the HR and RR percentiles they developed were very similar to those reported in the 2013 study, ${ }^{7}$ which used data from 2 other institutions, externally validating their findings.

The team then pushed the data a step further in a safety analysis and evaluated the sensitivity of the 5th and 95th percentiles for HR and RR from this study for detecting deterioration in 148 patients in the 12 hours before either a rapid response team activation or a cardiorespiratory arrest. The overall sensitivity for having either a HR or RR value out of range was 93\% for Goel and colleagues' percentiles and 97\% for the NIH ranges. Goel and colleagues concluded that using the 5th and 95th HR and RR percentiles provides a potentially safe means by which to modify physiologic bedside monitor alarm limits.

There are 2 important limitations to this work. The first is that the study uses EHR-documented data to estimate the performance of new physiologic monitor settings. Although there are few published reports of differences between nurse-charted vital signs and monitor data, those that do exist suggest that nurse charting favors more stable vital signs, ${ }^{9,10}$ even when charting oxygen saturation in patients with true, prolonged desaturation. ${ }^{9}$ We agree with the authors of 1 report, who speculated that nurses "recognize that temporary changes in vital signs are untypical for that patient and might choose to ignore them and either await a period of stability or make an educated estimate for that hour." 9 When using Goel and colleagues' 5th and 95th percentiles as alarm parameters, the expected scenario is that monitors will generate alarms for $10 \%$ of HR values and $10 \%$ of RR values. Because of the differences between nurse-charted vital 
signs and monitor data, the monitors will probably generate many more alarms.

The second limitation is the approach Goel and colleagues took in performing a safety analysis using chart review. Unfortunately, it is nearly impossible for a retrospective chart review to form the basis of a convincing scientific argument for the safety of different alarm parameters. It requires balancing the complex and sometimes competing nurse-level, patient-level, and alarm-level factors that determine nurse response time to alarms. It is possible to do prospectively, and we hope Goel's team will follow up this article with a description of the implementation and safety of these parameters in clinical practice.

In addition, the clinical implications of $\mathrm{HR}$ and RR at the 95th percentile might be considered less immediately life threatening than HR and RR at the 5th percentile, even though statistically they are equally abnormal. When choosing percentile-based alarm parameters, statistical symmetry might be less important than the potential immediate consequences of missing bradycardia or bradypnea. It would be reasonable to consider setting high $\mathrm{HR}$ and $\mathrm{RR}$ at the 99th percentile or higher, because elevated HR or RR alone is rarely immediately actionable, and set the low $\mathrm{HR}$ and RR at the 5 th or 10 th percentile.

Despite these caveats, should the percentiles proposed by Goel and colleagues be used to inform pediatric vital sign clinical decision support throughout the world? When faced with the alternative of using vital sign parameters that are not based on data from hospitalized children, these percentiles offer a clear advantage, especially for hospitals similar to Goel's. The most obvious immediate use for these percentiles is to improve noninterruptive ${ }^{11}$ vital sign clinical decision support in the EHR, the actual source of the data in this study.

The question of whether to implement Goel's 5th and 95th percentiles as physiologic monitor alarm parameters is more complex. In contrast to EHR decision support, there are much clearer downstream consequences of sounding unnecessary alarms as well as failing to sound important alarms for a child in extremis. Because their percentiles are not based on monitor data, the projected number of alarms generated at different percentile thresholds cannot be accurately estimated, although using their 5th and 95th percentiles should result in fewer alarms than the NIH parameters.
In conclusion, the work by Goel and colleagues represents an important contribution to knowledge about the ranges of expected vital signs in hospitalized children. Their findings can be immediately used to guide EHR decision support. Their percentiles are also relevant to physiologic monitor alarm parameters, although the performance and safety of using the 5th and 95th percentiles remain in question. Hospitals aiming to implement these data-driven parameters should first evaluate the performance of different percentiles from this article using data obtained from their own monitor system and, if proceeding with clinical implementation, pilot the parameters to accurately gauge alarm rates and assess safety before spreading hospital wide.

\section{Disclosures}

Dr. Bonafide is supported by a Mentored Patient-Oriented Research Career Development Award from the National Heart, Lung, and Blood Institute of the National Institutes of Health under award number K23HL116427. Dr. Brady is supported by a Patient-Centered Outcomes Research Mentored Clinical Investigator Award from the Agency for Healthcare Research and Quality under award number K08HS023827. The content is solely the responsibility of the authors and does not necessarily represent the official views of the funding organizations. The funding organizations had no role in the design, preparation, review, or approval of this article; nor the decision to submit the article for publication. The authors have no financial relationships relevant to this article or conflicts of interest to disclose.

\section{References}

1. Goel VV, Poole SF, Longhurst CA, et al. Safety analysis of proposed data-driven physiologic alarm parameters for hospitalized children. J Hosp Med. 2016;11(12):817-823.

2. Bonafide CP, Lin R, Zander M, et al. Association between exposure to nonactionable physiologic monitor alarms and response time in a children's hospital. J Hosp Med. 2015;10(6):345-351.

3. Lawless ST. Crying wolf: false alarms in a pediatric intensive care unit. Crit Care Med. 1994;22(6):981-985.

4. Rosman EC, Blaufox AD, Menco A, Trope R, Seiden HS. What are we missing? Arrhythmia detection in the pediatric intensive care unit. J Pediatr. 2013;163(2):511-514.

5. Talley LB, Hooper J, Jacobs B, et al. Cardiopulmonary monitors and clinically significant events in critically ill children. Biomed Instrum Technol. 2011;(suppl):38-45.

6. Tsien CL, Fackler JC. Poor prognosis for existing monitors in the intensive care unit. Crit Care Med. 1997;25(4):614-619.

7. Bonafide CP, Brady PW, Keren R, Conway PH, Marsolo K, Daymont C. Development of heart and respiratory rate percentile curves for hospitalized children. Pediatrics. 2013;131:e1150-e1157.

8. NIH Clinical Center. Pediatric services: age-appropriate vital signs. Available at: https://web.archive.org/web/20041101222327/http://www.cc. nih.gov/ccc/pedweb/pedsstaff/age.html. Published November 1, 2004. Accessed June 9, 2016.

9. Taenzer AH, Pyke J, Herrick MD, Dodds TM, McGrath SP. A comparison of oxygen saturation data in inpatients with low oxygen saturation using automated continuous monitoring and intermittent manual data charting. Anesth Analg. 2014;118(2):326-331.

10. Cunningham S, Deere S, Elton RA, McIntosh N. Comparison of nurse and computer charting of physiological variables in an intensive care unit. Int J Clin Monit Comput. 1996;13(4):235-241.

11. Phansalkar S, Sijs H van der, Tucker AD, et al. Drug-drug interactions that should be non-interruptive in order to reduce alert fatigue in electronic health records. J Am Med Inform Assoc. 2013;20(3):489-493. 